\title{
@creative
}

ISSN 1855-3966 (printed edn.), ISSN 1855-3974 (electronic edn.)

ARS MATHEMATICA CONTEMPORANEA 22 (2022) \#P2.08

https://doi.org/10.26493/1855-3974.2584.68d

(Also available at http://amc-journal.eu)

\section{The antiprism of an abstract polytope*}

\author{
Ian Gleason \\ Univeristy of California, Berkeley, United States \\ Isabel Hubard \\ Instituto de Matemáticas, Universidad Nacional Autonoma de México, \\ Circuito Exterior, C.U. Coyoacán 04510, México D.F.
}

Received 21 March 2021, accepted 19 August 2021, published online 14 June 2022

\begin{abstract}
Antiprisms of polygons are classical convex vertex-transitive polyhedra. In this paper, for any given (abstract) polytope, we define its antiprism. We then find the automorphism group of the antiprism of $\mathcal{P}$ in terms of the extended group of $\mathcal{P}$ (the groups of automorphisms and dualities) as well as some transitivity properties. We also give a relation between some products of abstract polytopes and their antiprisms.
\end{abstract}

Keywords: Antiprism, abstact polytopes.

Math. Subj. Class. (2020): 51M20, 52B05

The antiprism is a classical convex polyhedron. The antiprism of a polygon can be constructed by taking, in Euclidian 3 -space, two identical copies of a regular $n$-gon in parallel planes, in such a way that the vertices of one of the polygons are "aligned" with the mid points of the edges of the other. By taking the convex hull of all the vertices, we obtain the antiprism over an $n$-gon (see Figure 1 ).

For higher dimensions, the concept of a convex antiprism is not always defined (see [1, 2] and [3] for further discussion of the subject). In this paper we define the antiprism of any abstract polytope and show that it is indeed again an abstract polytope. The given definition generalizes the antiprism of a polygon and satisfies that the antiprism of a polytope and its dual is the same.

The paper uses some of the ideas and notation of the products of polytopes described in [4]. Moreover, we give relations between some products and their antiprisms. We then use such relations to compute the automorphism group of an antiprism. These results are summarized in the following theorem.

* The authors would like to thank Steve Wilson for suggesting studying the antiprism of an abstract polytope. We also gratefully acknowledge financial support of the PAPIIT-DGAPA, under grant IN-109218 and of CONACyT, under grant A1-S-21678.

†Corresponding author.

E-mail addresses: ianandreigf@gmail.com (Ian Gleason), isahubard@im.unam.mx (Isabel Hubard) 


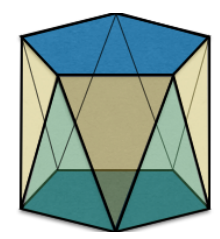

Figure 1: Antiprism over a pentagon.

Theorem A. Let $\mathcal{P}$ and $\mathcal{Q}$ be two abstract polytopes and $\operatorname{Ant}(\mathcal{P}), \operatorname{Ant}(\mathcal{Q})$ be their antiprisms.

(a) If $\bowtie$ and $\oplus$ denote the join product and the direct sum of abstract polytopes, respectively, then $\operatorname{Ant}(\mathcal{P} \bowtie \mathcal{Q}) \cong \operatorname{Ant}(\mathcal{P}) \oplus \operatorname{Ant}(\mathcal{Q})$.

(b) If $\hat{\Gamma}$ denotes the extended group of $\mathcal{P}$ and $\mathcal{P}=\mathcal{Q}_{1}^{m_{1}} \bowtie \mathcal{Q}_{2}^{m_{2}} \bowtie \cdots \bowtie \mathcal{Q}_{r}^{m_{r}}$, where each $\mathcal{Q}_{i}$ is a prime polytope with respect to the join product, then

$$
\Gamma(A n t(\mathcal{P}))=\Pi_{i=1}^{r}\left(\left(\hat{\Gamma}\left(\mathcal{Q}_{i}\right)\right)^{m_{i}} \rtimes S_{m_{i}}\right) .
$$

In particular, if $\mathcal{P}$ is prime with respect to the join product, then $\Gamma(A n t(\mathcal{P})) \cong \Gamma(\mathcal{P})$ whenever $\mathcal{P}$ is a not a self-dual polytope, while if $\mathcal{P}$ is self-dual, then $\Gamma(\mathcal{P})$ has index 2 in $\Gamma(\operatorname{Ant}(\mathcal{P}))=\hat{\Gamma}(\mathcal{P})$.

(c) Let $\mathcal{P}$ be a prime polytope with respect to the join product. If $\mathcal{P}$ is a $k$-orbit polytope of rank $n$, then $\operatorname{Ant}(\mathcal{P})$ is either a $2^{n} k$-orbit polytope (if $\mathcal{P}$ is self-dual) or $a 2^{n+1} k$ orbit polytope (if $\mathcal{P}$ is not self-dual).

The paper is organized as follows. Section 1 reviews the basic notions about abstract polytopes and their products. In Section 2 we define the antiprism and show that is always an abstract polytope and analyse the flags of the antiprism in terms of the flags of the polytope. Sections 3 and 4 deal with the interaction between some products and the antiprism, and with the study of the automorphism group of an antiprism, respectively.

\section{Abstract polytopes, their join product and direct sum}

Abstract polytopes are combinatorial generalizations of the face lattice of convex polytopes. In this section we give the basic definitions from the theory of abstract polytopes, as well as two of their products. For details on these subjects we refer the reader to [5] and [4], respectively.

An (abstract) polytope is a partially ordered set (poset) $\mathcal{P}$, whose elements are called faces, such that it has a minimal and a maximal element and is ranked: all its maximal chains, called flags, have the same number of elements. This endows the poset with a rank function $r$ satisfying that if $F, G \in \mathcal{P}$ with $F \leq G$, then $r(F) \leq r(G)$, and if $r(F)=r(G)$, then $F$ and $G$ are either equal or they are not incident in $\mathcal{P}$. We say that the minimal face has rank -1 , and if the range of the rank function is $\{-1,0, \ldots, n\}$, then we say that $\mathcal{P}$ has rank $n$ or is a $n$-polytope. A face of rank $i$ is said to be an $i$-face and the $0-, 1$ - and $n-1$-faces are the vertices, edges and facets of $\mathcal{P}$, respectively. The minimal and maximal faces are the improper faces of $\mathcal{P}$, and all other faces are proper. We also require that $\mathcal{P}$ satisfies the diamond condition, meaning that whenever $F, G \in \mathcal{P}$ are two incident faces 
such that their ranks differ by 2 , then there are exactly two faces $H, H^{\prime}$ of $\mathcal{P}$ satisfying that $F<H, H^{\prime}<G$. Finally, we ask that $\mathcal{P}$ be strongly connected in the sense that the poset is connected and each of its open intervals with more than two elements is connected as well.

A section of $\mathcal{P}$ is a closed interval of $\mathcal{P}$. Every section of $\mathcal{P}$ is a polytope in its own right. The diamond condition is equivalent to saying that all sections of rank 1 have exactly 4 faces. This condition also implies that for each $i \in\{0,1, \ldots, n-1\}$ and every flag $\Phi$, there is a unique $i$-adjacent flag to $\Phi$ that differs from $\Phi$ only in the element of rank $i$. We shall denote the set of all flags of $\mathcal{P}$ by $\mathcal{F}(\mathcal{P})$, and the $i$-adjacent flag of $\Phi$ by $\Phi^{i}$.

The dual of a polytope $\mathcal{P}$ is the poset that has the same elements as $\mathcal{P}$, but with the reverse order. If a polytope is isomorphic to its dual, it is said to be self-dual.

An automorphism of $\mathcal{P}$ is an order preserving bijection. The group of all automorphisms of $\mathcal{P}$ is its automorphism group and it shall be denoted by $\Gamma(\mathcal{P})$. A duality of a self-dual polytope is an order reversing bijection. The composition of two dualities of a self-dual polytope is not a duality, but an automorphim. Thus, the extended group of $\mathcal{P}$ is the group that contains all automorphisms and dualities of $\mathcal{P}$ and it will be denoted by $\hat{\Gamma}(\mathcal{P})$. Note that $\hat{\Gamma}(\mathcal{P})$ has $\Gamma(\mathcal{P})$ as a subgroup of index at most 2 ; the groups coincide whenever $\mathcal{P}$ is not self-dual.

Given two polytopes $\mathcal{P}$ and $\mathcal{Q}$, their join product, $\mathcal{P} \bowtie \mathcal{Q}$, is the polytope whose elements are the pairs $(F, G)$, with $F \in \mathcal{P}$ and $G \in \mathcal{Q}$. Two elements $(F, G)$ and $\left(F^{\prime}, G^{\prime}\right)$ are incident in $\mathcal{P} \bowtie \mathcal{Q}$ if and only if $F \leq_{\mathcal{P}} F^{\prime}$ and $G \leq_{\mathcal{Q}} G^{\prime}$. The rank of $(F, G)$ is $\operatorname{rank}_{\mathcal{P}}(F)+\operatorname{rank}_{\mathcal{Q}}(G)+1$. A polytope $\mathcal{P}$ is said to be prime with respect to the join product if it cannot be decomposed as the join product of two polytopes of ranks at least 0 .

The direct sum of the polytopes $\mathcal{P}$ and $\mathcal{Q}$, with maximum elements $F_{n}$ and $G_{m}$, respectively is $\mathcal{P} \oplus \mathcal{Q}=\left\{(F, G) \in \mathcal{P} \bowtie \mathcal{Q} \mid F \neq F_{n}, G \neq G_{m}\right\} \cup\left\{\left(F_{n}, G_{m}\right)\right\}$. The order of the direct sum is given by $(F, G) \leq_{\mathcal{P} \oplus \mathcal{Q}}\left(F^{\prime}, G^{\prime}\right)$ if and only if $F \leq_{\mathcal{P}} F^{\prime}$ and $G \leq_{\mathcal{Q}} G^{\prime}$, and the rank of the face $(F, G)$ is $\operatorname{rank}_{\mathcal{P}}(F)+\operatorname{rank}_{\mathcal{Q}}(G)$, which implies that the rank of $\mathcal{P} \oplus \mathcal{Q}$ is $n+m$. A polytope $\mathcal{P}$ is said to be prime with respect to the direct sum if it cannot be decomposed as the join product of two polytopes of ranks at least 1 . The following lemma falls straightforward from the definitions.

Lemma 1.1. Let $\mathcal{P}$ and $\mathcal{Q}$ be two polytopes, and let $(F, G)$ be a proper face of $\mathcal{P} \oplus \mathcal{Q}$. Then the section $\left\{(H, K) \in \mathcal{P} \oplus \mathcal{Q} \mid(H, K) \leq_{\mathcal{P} \oplus \mathcal{Q}}(F, G)\right\}$ is isomorphic to the join product of the sections $\left\{H \in \mathcal{P} \mid H \leq_{\mathcal{P}} F\right\}$ and $\left\{K \in \mathcal{Q} \mid K \leq_{\mathcal{Q}} G\right\}$.

In [4] the authors study the automorphism group of a product in terms of the automorphisms groups of the factors. In particular we have the following result.

Theorem $1.2([4])$. Let $\mathcal{P}=\mathcal{Q}_{1}^{m_{1}} \oplus \mathcal{Q}_{2}^{m_{2}} \oplus \cdots \oplus \mathcal{Q}_{r}^{m_{r}}$, where each $\mathcal{Q}_{i}$ is a prime polytope with respect to the direct sum. Then

$$
\Gamma(\mathcal{P})=\Pi_{i=1}^{r}\left(\left(\Gamma\left(\mathcal{Q}_{i}\right)\right)^{m_{i}} \rtimes S_{m_{i}}\right) .
$$

\section{The antiprism}

The antiprism of a polygon is a convex polyhedron in ordinary 3-space. Its faces are two regular $n$-gons and $2 n$ equilateral triangles. When $n=3$, we obtain the regular octahedron. Otherwise, the antiprism over an $n$-gon is an Archemidian solid, as their faces are all regular polygons and its group of symmetries acts transitively on the vertices. In this section, for each polytope $\mathcal{P}$, we give a construction of a new polytope $\operatorname{Ant}(\mathcal{P})$ which generalizes the construction of the antiprism of a polygon. 
Let $\mathcal{P}$ be an $n$-polytope. To formally define the antiprism of $\mathcal{P}, \operatorname{Ant}(\mathcal{P})$, we let $P$ be a symbol, and define

$$
\operatorname{Ant}(\mathcal{P}):=\left\{(F, G) \mid F, G \in \mathcal{P}, F \leq_{\mathcal{P}} G\right\} \cup\{P\}
$$

where the order is given by

$$
\begin{aligned}
& (F, G) \leq(H, K) \text { if and only if } F \leq_{\mathcal{P}} H \leq_{\mathcal{P}} K \leq_{\mathcal{P}} G \\
& (F, G) \leq P \quad \text { for every } F, G \in \mathcal{P} .
\end{aligned}
$$

Throughout this section, when we say that an ordered pair of elements of $\mathcal{P}$ is an element of $\operatorname{Ant}(\mathcal{P})$, we shall be referring to an element of $A n t(\mathcal{P})$ different than $P$.

Note then that $P$ is the maximum element of $A n t(\mathcal{P})$ and that, if $F_{-1}$ and $F_{n}$ denote the minimum and maximum elements of $\mathcal{P}$, respectively, then $\left(F_{-1}, F_{n}\right)$ is in fact the minimum element of $\operatorname{Ant}(\mathcal{P})$. Moreover, for $H, F, G \in \mathcal{P}$, with $H \leq_{\mathcal{P}} F \leq_{\mathcal{P}} G$, we have that $(H, F) \leq(F, F)$ and $(F, G) \leq(F, F)$, but the only element of $\operatorname{Ant}(\mathcal{P})$ greater than $(F, F)$ is $P$.

Suppose that $\mathcal{P}$ has rank $n$ and its rank function is $\operatorname{rank}_{\mathcal{P}}$. Define

$$
\begin{aligned}
\operatorname{rank}_{A n t(\mathcal{P})}(F, G) & :=\operatorname{rank}(F, G)=n+\operatorname{rank}_{\mathcal{P}}(F)-\operatorname{rank}_{\mathcal{P}}(G), \\
\operatorname{rank}_{A n t(\mathcal{P})}(P) & :=\operatorname{rank}(P)=n+1 .
\end{aligned}
$$

Note that for every $(F, G) \in \operatorname{Ant}(\mathcal{P})$, we have that $0 \leq \operatorname{rank}_{\mathcal{P}}(G)-\operatorname{rank}_{\mathcal{P}}(F) \leq n+$ 1 , implying that $\operatorname{rank}(F, G) \in\{-1, \ldots, n\}$ and therefore rank: $\operatorname{Ant}(\mathcal{P}) \rightarrow\{-1, \ldots$, $n+1\}$.

Moreover, if $\operatorname{rank}(F, G)=-1$, then

$$
n+\operatorname{rank}_{\mathcal{P}}(F)-\operatorname{rank}_{\mathcal{P}}(G)=-1 .
$$

This is equivalent to have that

$$
\operatorname{rank}_{\mathcal{P}}(G)=n+1+\operatorname{rank}_{\mathcal{P}}(F) .
$$

But $\operatorname{rank}_{\mathcal{P}}(G) \leq n$ which implies that $F$ should have rank -1 , and thus $G$ has rank $n$; in other words, $\operatorname{rank}(F, G)=-1$ if and only if $F=F_{-1}$ and $G=F_{n}$. We can further see that $\operatorname{rank}(F, G)=n$ if and only if $F=G$. Hence, the facets of $\operatorname{Ant}(\mathcal{P})$ are the elements $(F, F)$, with $F \in \mathcal{P}$.

There are other faces of $\operatorname{Ant}(\mathcal{P})$ that are easy to identify. For example, if $(F, G)$ is a vertex, it should satisfy that $\operatorname{rank}(F, G)=n+\operatorname{rank}_{\mathcal{P}}(F)-\operatorname{rank}_{\mathcal{P}}(G)=0$. That is,

$$
\operatorname{rank}_{\mathcal{P}}(G)=n+\operatorname{rank}_{\mathcal{P}}(F) .
$$

Again, $\operatorname{since}_{\operatorname{rank}_{\mathcal{P}}}(G) \leq n$ we have two options: either $\operatorname{rank}_{\mathcal{P}}(G)=n$ and $\operatorname{rank}_{\mathcal{P}}(F)=$ 0 , or $\operatorname{rank}_{\mathcal{P}}(G)=n-1$ and $\operatorname{rank}_{\mathcal{P}}(F)=F_{-1}$. This implies that the vertices of $\operatorname{Ant}(\mathcal{P})$ are either of the form $\left(v, F_{n}\right)$, where $v$ is a vertex of $\mathcal{P}$, or of the form $\left(F_{-1}, f\right)$, where $f$ is a facet of $\mathcal{P}$.

Before showing that $\operatorname{Ant}(\mathcal{P})$ is a polytope, let us analyze the case when $\mathcal{P}$ is 2 polytope. Let $\mathcal{P}$ be a 2 -polytope with vertices $\left\{v_{1}, \ldots, v_{p}\right\}$ and edges $\left\{e_{1}, \ldots e_{p}\right\}$ in such a way that for every $i=1, \ldots, p-1, v_{i}, v_{i+1} \leq e_{i}$, and $v_{1}, v_{p} \leq e_{p}$. Let $m$ and $M$ be the 
minimum and maximum elements of $\mathcal{P}$, respectively. We already know that $\operatorname{Ant}(\mathcal{P})$ has a unique minimum $(m, M)$ and a unique maximum $P$, that there are $2 p$ vertices, namely:

$$
\left(v_{1}, M\right), \ldots,\left(v_{p}, M\right),\left(m, e_{1}\right), \ldots,\left(m, e_{p}\right),
$$

and that the facets, 2 -faces in this case, are of the form $(F, F)$, where $F$ is any element of $\mathcal{P}$. Thus there are $2 p+2$ facets. Finally, the 1 -faces are the elements

$$
\begin{array}{r}
\left(e_{1}, M\right), \ldots,\left(e_{p}, M\right), \\
\left(m, v_{1}\right), \ldots,\left(m, v_{p}\right), \\
\left(v_{1}, e_{p}\right),\left(v_{1}, e_{1}\right),\left(v_{2}, e_{1}\right), \ldots,\left(v_{p}, e_{p}\right),
\end{array}
$$

and there are $4 p$ of them.

We note that the facets $(m, m)$ and $(M, M)$ are $p$-gons, as their vertices are of the form $\left(m, e_{i}\right)$ and $\left(v_{i}, M\right)$, respectively. In contrast, the facets of type $\left(v_{i}, v_{i}\right)$ and $\left(e_{i}, e_{i}\right)$ are triangles, as their only vertices are either of the form $\left(v_{i}, M\right),\left(m, e_{i-1}\right),\left(m, e_{i}\right)$ or of the form $\left(v_{i}, M\right),\left(v_{i+1}, M\right),\left(m, e_{i}\right)$. It is not too difficult now to see that $\operatorname{Ant}(\mathcal{P})$ is in fact isomorphic to the classical antiprism.

Given an abstract polytope $\mathcal{P}$, we should say that $\operatorname{Ant}(\mathcal{P})$ is the antiprism of $\mathcal{P}$.

In order to show that the antiprism of any polytope is again a polytope, we shall start by analyzing the sections of $\operatorname{Ant}(\mathcal{P})$. As we noted before, the only elements of rank $n$ are of the type $(F, F)$, where $F \in \mathcal{P}$. Let us take a look into the sections $\mathcal{Q}_{F}:=$ $(F, F) /\left(F_{-1}, F_{n}\right)$ where, as before, $F_{-1}, F_{n}$ are the minimum and maximum faces of the $n$-polytope $\mathcal{P}$, respectively.

Let us fix a face $F$ of $\mathcal{P}$. If $(H, G) \in \mathcal{Q}_{F}$, then $(H, G) \leq(F, F)$, which implies that $H \leq F \leq F \leq G$. In other words, $F$ is a face of the section $G / H$ of $\mathcal{P}$. On the other hand, if $H, G \in \mathcal{P}$ are such that $H \leq F$ and $F \leq G$, then $(H, G) \in \mathcal{Q}_{F}$. That means that the faces of the section $\mathcal{Q}_{F}$ are in one to one correspondence with the order pairs $(H, G)$ of elements of $\mathcal{P}$ such that $H \leq F \leq G$.

Since $\mathcal{P}$ is a polytope, then $\mathcal{P}_{F}^{-}:=F / F_{-1}$ and $\mathcal{P}_{F}^{+}:=F_{n} / F$ are also polytopes. Let $\delta: \mathcal{P}_{F}^{+} \rightarrow\left(\mathcal{P}_{F}^{+}\right)^{*}$ be a duality mapping $\mathcal{P}_{F}^{+}$to its dual. Now, $H \in \mathcal{P}_{F}^{-}$if and only if $H \leq F$; on the other hand, $F \leq G$ if and only if $G \delta \in\left(\mathcal{P}_{F}^{+}\right)^{*}$. Consider now the join product of $\mathcal{P}_{F}^{-}$with $\left(\mathcal{P}_{F}^{+}\right)^{*}$. We have that

$$
\begin{aligned}
\psi: \mathcal{P}_{F}^{-} \bowtie\left(\mathcal{P}_{F}^{+}\right)^{*} & \rightarrow \mathcal{Q}_{F} \\
(H, G \delta) & \mapsto(H, G)
\end{aligned}
$$

is a well-defined bijection between $\mathcal{P}_{F}^{-} \bowtie\left(\mathcal{P}_{F}^{+}\right)^{*}$ and $\mathcal{Q}_{F}$. Furthermore, note that $(H, G \delta) \leq_{\bowtie}\left(H^{\prime}, G^{\prime} \delta\right)$ if and only if $H \leq H^{\prime}$ and $G \delta \leq G^{\prime} \delta$, which is equivalent to have $H \leq H^{\prime}$ and $G^{\prime} \leq G$. That is, $(H, G \delta) \leq_{\bowtie}\left(H^{\prime}, G^{\prime} \delta\right)$ if and only if $H \leq H^{\prime} \leq$ $F \leq G^{\prime} \leq G$, which is equivalent to have that $(H, G) \leq_{\mathcal{Q}_{F}}\left(H^{\prime}, G^{\prime}\right)$. Thus, $\psi$ is an isomorphism between $\mathcal{P}_{F}^{-} \bowtie\left(\mathcal{P}_{F}^{+}\right)^{*}$ and $\mathcal{Q}_{F}$. This implies that all the facets of $\operatorname{Ant}(\mathcal{P})$ are abstract polytopes. In particular we note that $\mathcal{Q}_{F_{-1}} \cong \mathcal{P}^{*}$, while $\mathcal{Q}_{F_{n}} \cong \mathcal{P}$.

We turn now our attention to the co-faces $P /(F, G)$ of $\operatorname{Ant}(\mathcal{P})$. We observe that

$$
\begin{aligned}
P /(F, G) & =\left\{(H, K) \in \operatorname{Ant}(\mathcal{P}) \mid(F, G) \leq_{\text {Ant }(\mathcal{P})}(H, K)\right\} \cup\{P\} \\
& \left.\cong\left\{(H, K) \in \mathcal{P} * \mathcal{P} \mid F \leq_{\mathcal{P}} H \leq_{\mathcal{P}} K \leq_{\mathcal{P}} G\right)\right\} \cup\{P\} \\
& \cong\left\{(H, K) \in \mathcal{P} * \mathcal{P} \mid H, K \in G / F, H \leq_{G / F} K\right\} \cup\{P\} \\
& \cong \operatorname{Ant}(G / F) .
\end{aligned}
$$


This says that all the co-faces of $\operatorname{Ant}(\mathcal{P})$ are antiprisms of polytopes of smaller rank than that of $\mathcal{P}$.

Theorem 2.1. Let $\mathcal{P}$ be an $n$-polytope, then $\operatorname{Ant}(\mathcal{P})$ is an $n+1$ polytope.

Proof. The function given in (2.3) is the desired rank function, with range $\{-1, \ldots, n+1\}$, and it is clear from the definition that $\operatorname{Ant}(\mathcal{P})$ has a minimum and a maximum face.

We now proceed by induction over $n$.

Let $\mathcal{P}=\left\{F_{-1}, F_{0}\right\}$ be a 0-polytope. Then $\operatorname{Ant}(\mathcal{P})=\left\{\left(F_{-1}, F_{0}\right),\left(F_{-1}, F_{-1}\right)\right.$, $\left.\left(F_{0}, F_{0}\right), P\right\}$, where $\left(F_{-1}, F_{0}\right) \leq\left(F_{-1}, F_{-1}\right),\left(F_{0}, F_{0}\right) \leq P$. Hence, $\operatorname{Ant}(\mathcal{P})$ is isomorphic to an edge, that is, $\operatorname{Ant}(\mathcal{P})$ is a 1-polytope.

Suppose now that the antiprism of any polytope of rank $(n-1)$ is a polytope and let $\mathcal{P}$ be an $n$-polytope. Since the facets of $\operatorname{Ant}(\mathcal{P})$ are a join product of polytopes, then they are polytopes. In particular, every flag of $\operatorname{Ant}(\mathcal{P})$, when taking away the maximum face, can be seen to be contained flag of a facet of $\operatorname{Ant}(\mathcal{P})$. Since the flags of the facets have all $n+2$ elements, every flag of $A n t(\mathcal{P})$ has exactly $n+3$ elements.

The diamond condition is satisfied and all the proper sections of $\operatorname{Ant}(\mathcal{P})$ are connected: this is straightforward to see as a proper section of $\operatorname{Ant}(\mathcal{P})$ is contained either in a facet or in a vertex figure of $A n t(\mathcal{P})$. The facets of $A n t(\mathcal{P})$ are joins of polytopes (hence polytopes) and the vertex figures are anitprisms over proper sections of $\mathcal{P}$, which by hypothesis of induction are also polytopes.

We only have to see that $\operatorname{Ant}(\mathcal{P})$ itself is connected. Let $(F, G),(H, K)$ be two proper faces of $\operatorname{Ant}(\mathcal{P})$. We divide the analysis in several cases, depending on whether or not $F, G, H$ and $K$ are proper or improper faces of $\mathcal{P}$. Note that $F$ and $G$ (resp. $H$ and $K$ ) cannot be improper face of $\mathcal{P}$ simultaneously, unless they are equal. Without loss of generality, we may assume that $\operatorname{rank}_{\mathcal{P}}(G) \leq \operatorname{rank}_{\mathcal{P}}(K)$.

- If $G, K \neq F_{n}$, then $(F, G),\left(F_{-1}, G\right),\left(F_{-1}, F_{-1}\right),\left(F_{-1}, K\right),(H, K)$ is a sequence of incident proper faces of $\operatorname{Ant}(\mathcal{P})$.

- If $F, H \neq F_{-1}$, then $(F, G),\left(F, F_{n}\right),\left(F_{n}, F_{n}\right),\left(H, F_{n}\right),(H, K)$ is a sequence of incident proper faces of $\operatorname{Ant}(\mathcal{P})$.

- If $K=F_{n}, F=F_{-1}$ and $H$ is a proper face of $\mathcal{P}$, then $H \neq F_{-1}, F_{n}$ and $G \neq$ $F_{n}$. Since $\mathcal{P}$ is connected, then there exists a sequence $G=G_{1}, G_{2}, \ldots, G_{h}=H$ of incident faces of $\mathcal{P}$ all of which, except perhaps for $G$, are proper faces. Then $(F, G)=\left(F_{-1}, G_{1}\right),\left(F_{-1}, G_{2}\right), \ldots,\left(F_{-1}, G_{h}\right)=\left(F_{-1}, H\right),(H, H),\left(H, F_{n}\right)=$ $(H, K)$ is a sequence of incident proper faces of $\operatorname{Ant}(\mathcal{P})$.

- If $K=H=F_{n}, F=F_{-1}$ and $G$ is a proper face of $\mathcal{P}$, then $\left(F_{-1}, G\right),(G, G)$, $\left(G, F_{n}\right),\left(F_{n}, F_{n}\right)$ is a sequence of incident proper faces of $\operatorname{Ant}(\mathcal{P})$.

- If $K=H=F_{n}, F=G=F_{-1}$, then let $J \in \mathcal{P}$ be any proper face of $\mathcal{P}$ (exists as we are assuming $n>0)$. Hence $\left(F_{-1}, F_{-1}\right),\left(F_{-1}, J\right),(J, J),\left(J, F_{n}\right),\left(F_{n}, F_{n}\right)$ is a sequence of incident proper faces of $\operatorname{Ant}(\mathcal{P})$.

Hence $\operatorname{Ant}(\mathcal{P})$ is connected and therefore it is a polytope.

Note that given a polytope $\mathcal{P}$ and its dual $\mathcal{P}^{*}$, there is a duality $\delta: \mathcal{P} \rightarrow \mathcal{P}^{*}$. We know that $\delta$ is a bijection that reverses the order, and hence every element of $\mathcal{P}^{*}$ can be written as $F \delta$, where $F$ is a face of $\mathcal{P}$. Hence, there is a natural bijection between the faces of 
the antirpism of $\mathcal{P}$ and the faces of the antiprism of $\mathcal{P}^{*}$. In fact, we have the following proposition.

Proposition 2.2. For any polytope $\mathcal{P}, \operatorname{Ant}(\mathcal{P}) \cong A n t\left(\mathcal{P}^{*}\right)$, where $\mathcal{P}^{*}$ denotes the dual of $\mathcal{P}$.

Proof. Let $P$ and $P^{*}$ be the maximum elements of $\operatorname{Ant}(\mathcal{P})$ and $\operatorname{Ant}\left(\mathcal{P}^{*}\right)$, respectively, and let $\delta: \mathcal{P} \rightarrow \mathcal{P}^{*}$ be a duality. Let $\psi: \operatorname{Ant}(\mathcal{P}) \rightarrow \operatorname{Ant}\left(\mathcal{P}^{*}\right)$ be given by:

$$
\begin{aligned}
(F, G) & \mapsto(G \delta, F \delta), \\
P & \mapsto P^{*}
\end{aligned}
$$

Then clearly $\psi$ is a well-defined bijection between $\operatorname{Ant}(\mathcal{P})$ and $\operatorname{Ant}\left(\mathcal{P}^{*}\right)$. Furthermore $(F, G) \leq_{A n t(\mathcal{P})}(H, K)$ if and only if $F \leq_{\mathcal{P}} H \leq_{\mathcal{P}} K \leq_{\mathcal{P}} G$ if and only if $G \delta \leq_{\mathcal{P}^{*}}$ $K \delta \leq_{\mathcal{P}^{*}} H \delta \leq_{\mathcal{P}^{*}} F \delta$ if and only if $(G \delta, F \delta) \leq_{\mathcal{P}^{*}}(K \delta, H \delta)$ which is equivalent to $(F, G) \psi \leq_{\mathcal{P}^{*}}(H, K) \psi$. Since it is now straightforward to see that $\delta^{-1}$ also induces a bijection that preserves the order and is the inverse of $\psi$. This settles the proposition.

\subsection{The flags of a polytope and the flags of its antiprism}

In this section we study the relation between the flags of $A n t(\mathcal{P})$ and the flags of $\mathcal{P}$.

Let $\mathcal{P}$ be an $n$-polytope (with maximum element $F_{n}$ and minimum element $F_{-1}$ ) and consider $V$ to be the set of all ordered $(n+1)$-tuples with entries 0 and 1 . We are going to see that there is a bijection between $\mathcal{F}(A n t(\mathcal{P}))$ and $\mathcal{F}(\mathcal{P}) \times V$. For this, consider a flag of $\operatorname{Ant}(\mathcal{P}),\left\{A_{-1}, A_{0}, \ldots A_{n+1}\right\}$, where $\operatorname{rank} A_{i}=i$. Then $A_{n+1}=P$ and for each $i=-1,0 \ldots n$, there exist $F^{i}, G^{i} \in \mathcal{P}$ such that $A_{i}=\left(F^{i}, G^{i}\right)$. It is straightforward to see that $F^{-1}=F_{-1}, G^{-1}=F_{n}$ and $F^{n}=G^{n}:=F$, for some $F \in \mathcal{P}$. Furthermore, observe that

$$
F_{-1} \leq F^{0} \leq F^{1} \leq \cdots \leq F^{n}=F=G^{n} \leq G^{n-1} \leq \cdots \leq G^{0} \leq G^{-1}=F_{n},
$$

is a sequence of faces of $\mathcal{P}$ in which, of course, many of the elements might repeat. For example, the sequence could be such that $F^{0}=F^{1}=F^{2}=\cdots=F^{n}=F_{-1}$.

On one hand, note that for a given $i \in\{0, \ldots n\}$, we have that either

$$
\operatorname{rank}\left(F^{i}\right)=\operatorname{rank}\left(F^{i+1}\right) \text { and } \operatorname{rank}\left(G^{i}\right)=\operatorname{rank}\left(G^{i+1}\right)+1
$$

or

$$
\operatorname{rank}\left(F^{i}\right)+1=\operatorname{rank}\left(F^{i+1}\right) \text { and } \operatorname{rank}\left(G^{i}\right)=\operatorname{rank}\left(G^{i+1}\right) .
$$

In particular, either $\operatorname{rank}\left(F^{0}\right)=-1$ and $\operatorname{rank}\left(G^{0}\right)=n-1$ or $\operatorname{rank}\left(F^{0}\right)=0$ and $\operatorname{rank}\left(G^{0}\right)=n$. Hence, we can regard the sequence in (2.5) as a sequence of incident faces of $\mathcal{P}$ that has exactly one element of each rank. That is, a flag of $\mathcal{P}$. In other words, each flag of $A n t(\mathcal{P})$ induces a flag of $\mathcal{P}$ in a natural way.

On the other hand, the sequence in (2.5) also defines an element of $V$ in the following way. For each $i \in\{0, \ldots, n\}$, let $a_{i}=0$ if $\operatorname{rank}\left(F^{i-1}\right)=\operatorname{rank}\left(F^{i}\right)$ and $a_{i}=1$ otherwise. It should be clear that $\left(a_{0}, \ldots a_{n}\right)$ is an element of $V$.

The above assignment is a bijection. To see this, take $\Phi \in \mathcal{F}(\mathcal{P})$ and $v \in V$. Denote by $\Phi_{i}$ the $i$-face of $\Phi$ and by $v_{i}$ the $i$-th element of $v$, i.e. $v=\left(v_{0}, v_{1}, \ldots, v_{n}\right)$. We define the flag $\left\{A_{-1}, A_{0}, \ldots A_{n+1}\right\}$ of $\operatorname{Ant}(\mathcal{P})$, where each $A_{i}=\left(F^{i}, G^{i}\right)$, in the following way. 
First, $A_{n+1}=P$ and $A_{-1}=\left(F_{-1}, F_{n}\right)=\left(\Phi_{-1}, \Phi_{n}\right)$. Now, we define inductively the elements $F^{i}$ and $G^{i}$. Suppose $F^{i-1}$ is defined as the $j$-face $\Phi_{j}$, then we define $F^{i}:=$ $\Phi_{j+v_{i}}$. (For example, if $v_{0}=0$, then $F^{0}=\Phi_{-1+v_{0}}=\Phi_{-1}=F_{-1}$, and if $v_{0}=1$, then $F^{0}=\Phi_{-1+v_{0}}=\Phi_{0}$.) Similarly, we first define $G^{n}:=F^{n}$ and, inductively, suppose that $G^{i+1}$ is defined as the $k$-face $\Phi_{k}$, then we define $G^{i}:=\Phi_{k+1-v_{i+1}}$. Thus, $\mid\left\{v_{j} \in\right.$ $\left.v \mid v_{j}=1\right\} \mid=m+1$, for some $-1 \leq m \leq n$ and hence $G^{n}=F^{n}=\Phi_{m}$ and $\left|\left\{v_{j} \in v \mid v_{j}=0\right\}\right|=n-m$. This implies that

$$
G^{-1}=\Phi_{m+\left(1-v_{n}\right)+\left(1-v_{n-1}+\cdots+\left(1-v_{0}\right)\right)}=\Phi_{m+(n-m)}=\Phi_{n} .
$$

It should not be difficult to see that this assignment of a flag of $\operatorname{Ant}(\mathcal{P})$, given a pair $(\Phi, v) \in \mathcal{F}(\mathcal{P}) \times V$ is inverse to the above description, where each flag of $\operatorname{Ant}(\mathcal{P})$ induces a flag of $\mathcal{P}$ and an element of $V$. We have therefore established that

Lemma 2.3. Let $\mathcal{P}$ be an n-polytope. Then the flags of $A n t(\mathcal{P})$ are in one-to-one correspondence with the set $\mathcal{F}(\mathcal{P}) \times V$, where $\mathcal{F}(\mathcal{P})$ denotes the set of flags of $\mathcal{P}$ and $V$ the set of all ordered $(n+1)$-tuples with entries 0 and 1 .

\section{Products and the antiprism}

In the next section we will study the automorphism group of an antiprism. We shall see that computing it for polytopes that are prime with respect to the join product is straighforward. To completely determine the automorphism group of any antiprism, we need some of the results given in this section.

All our results here deal with the interaction of the join product and the direct sum with the anitprism.

Proposition 3.1. Let $\mathcal{P}$ and $\mathcal{Q}$ be two polytopes. Then $\operatorname{Ant}(\mathcal{P} \bowtie \mathcal{Q}) \cong \operatorname{Ant}(\mathcal{P}) \oplus \operatorname{Ant}(\mathcal{Q})$.

Proof. Let $\psi: \operatorname{Ant}(\mathcal{P} \bowtie \mathcal{Q}) \rightarrow \operatorname{Ant}(\mathcal{P}) \oplus \operatorname{Ant}(\mathcal{Q})$ be such that

$$
((F, G),(H, K)) \psi=((F, H),(G, K))
$$

and, if $P, P_{\mathcal{P}}$ and $P_{\mathcal{Q}}$ are the maximum elements of $\operatorname{Ant}(\mathcal{P} \bowtie \mathcal{Q}), \operatorname{Ant}(\mathcal{P})$ and $\operatorname{Ant}(\mathcal{Q})$, respectively, then $P \psi=\left(P_{\mathcal{P}}, P_{\mathcal{Q}}\right)$. We shall show that $\psi$ is an isomorphism.

First note that $((F, G),(H, K)) \in \operatorname{Ant}(\mathcal{P} \bowtie \mathcal{Q})$ implies that $(F, G),(H, K) \in \mathcal{P} \bowtie \mathcal{Q}$ and that $(F, G) \leq_{\mathcal{P} \bowtie \mathcal{Q}}(H, K)$. Hence, we have that $F, H \in \mathcal{P}$ with $F \leq_{\mathcal{P}} H$, and that $G, K \in \mathcal{Q}$ with $G \leq_{\mathcal{Q}} K$; that is, $(F, H) \in \operatorname{Ant}(\mathcal{P})$ and $(G, K) \in \operatorname{Ant}(\mathcal{Q})$. Moreover, $(F, G)$ is not the maximum element of $\operatorname{Ant}(\mathcal{P})$, and $(H, K)$ is not the maximum element of $\operatorname{Ant}(\mathcal{Q})$, which implies that $((F, H),(G, K)) \in \operatorname{Ant}(\mathcal{P}) \oplus A n t(\mathcal{Q})$. Furthermore, observe that different elements of $\operatorname{Ant}(\mathcal{P} \bowtie \mathcal{Q})$ go to different elements of $\operatorname{Ant}(\mathcal{P}) \oplus \operatorname{Ant}(\mathcal{Q})$ under $\psi$ and therefore $\psi$ is a well-defined function from $\operatorname{Ant}(\mathcal{P} \bowtie \mathcal{Q})$ to $\operatorname{Ant}(\mathcal{P}) \oplus \operatorname{Ant}(\mathcal{Q})$.

Similarly, let $\phi: \operatorname{Ant}(\mathcal{P}) \oplus \operatorname{Ant}(\mathcal{Q}) \rightarrow \operatorname{Ant}(\mathcal{P} \bowtie \mathcal{Q})$ be such that

$$
((F, H),(G, K)) \mapsto((F, G),(H, K)) .
$$

A similar argument as the one above shows that $\phi$ is also a well-defined function. Note that both $\psi \phi$ and $\phi \psi$ are the identity map, which implies that both functions are bijections, and one is the inverse of the other. 
We need to show that these two functions preserve the orders. Let $\left(\left(F_{0}, G_{0}\right),\left(H_{0}, K_{0}\right)\right)$, $\left(\left(F_{1}, G_{1}\right),\left(H_{1}, K_{1}\right)\right) \in \operatorname{Ant}(\mathcal{P} \bowtie \mathcal{Q})$, then

$$
\begin{aligned}
& \left(\left(F_{0}, G_{0}\right),\left(H_{0}, K_{0}\right)\right) \leq_{A n t(\mathcal{P} \bowtie \mathcal{Q})}\left(\left(F_{1}, G_{1}\right),\left(H_{1}, K_{1}\right)\right) \\
& \Leftrightarrow\left(F_{0}, G_{0}\right) \leq_{\mathcal{P} \bowtie \mathcal{Q}}\left(F_{1}, G_{1}\right) \leq_{\mathcal{P} \bowtie \mathcal{Q}}\left(H_{1}, K_{1}\right) \leq_{\mathcal{P} \bowtie \mathcal{Q}}\left(H_{0}, K_{0}\right) \\
& \Leftrightarrow F_{0} \leq_{\mathcal{P}} F_{1} \leq_{\mathcal{P}} H_{1} \leq_{\mathcal{P}} H_{0} \text { and } G_{0} \leq_{\mathcal{Q}} G_{1} \leq_{\mathcal{Q}} K_{1} \leq_{\mathcal{Q}} K_{0} \\
& \Leftrightarrow\left(F_{0}, H_{0}\right) \leq_{\operatorname{Ant}(\mathcal{P})}\left(F_{1}, H_{1}\right) \text { and }\left(G_{0}, K_{0}\right) \leq_{\operatorname{Ant}(\mathcal{Q})}\left(G_{1}, K_{1}\right) \\
& \Leftrightarrow\left(\left(F_{0}, H_{0}\right),\left(G_{0}, K_{0}\right)\right) \leq_{\operatorname{Ant}(\mathcal{P}) \oplus \operatorname{Ant}(\mathcal{Q})}\left(\left(F_{1}, H_{1}\right),\left(G_{1}, K_{1}\right)\right) .
\end{aligned}
$$

Therefore both $\psi$ and $\phi$ preserve the orders and hence $\psi$ is an isomorphism.

Lemma 3.2. If $\mathcal{P}$ is a prime polytope with respect to the join product, then $\operatorname{Ant}(\mathcal{P})$ is a prime polytope with respect to the direct sum.

Proof. Suppose otherwise. Then there exists a polytope $\mathcal{P}$ that is prime with respect to the join product, but such that $\operatorname{Ant}(\mathcal{P})$ is not prime with respect to the direct sum. Let $\operatorname{Ant}(\mathcal{P})=\mathcal{Q} \oplus \mathcal{K}$, where $\mathcal{Q}$ and $\mathcal{K}$ are polytopes of rank at least 1 .

Note that $\operatorname{Ant}(\mathcal{P})$ contains a facet that is isomorphic to $\mathcal{P}$. In fact, if $F_{n}$ denotes the maximum element of $\mathcal{P}$, then $\left(F_{n}, F_{n}\right) \in \operatorname{Ant}(\mathcal{P})$ has rank $n$ and if $(F, G) \leq\left(F_{n}, F_{n}\right)$, then $G=F_{n}$ (since $F \leq_{\mathcal{P}} F_{n} \leq_{\mathcal{P}} F_{n} \leq_{\mathcal{P}} G$ ). That means that the section $\left(F_{n}, F_{n}\right) /\left(F_{-1}, F_{n}\right)$ of $\operatorname{Ant}(\mathcal{P})$ is isomorphic to $\mathcal{P}$.

But by Lemma 1.1, a facet of the direct product $\mathcal{Q} \oplus \mathcal{K}$ is isomorphic to a non-trivial join product. Hence $\mathcal{P}$ is not prime with respect to the join product, which contradicts our hypothesis.

\section{Automorphism groups}

We now turn our attention to the study of the automorphism group of the antiprism of $\mathcal{P}$.

It is not difficult to see that every automorphism of $\mathcal{P}$ induces an automorphism of $\operatorname{Ant}(\mathcal{P})$. In fact, given $\gamma \in \Gamma(\mathcal{P})$ the mapping $\hat{\gamma}: \operatorname{Ant}(\mathcal{P}) \rightarrow \operatorname{Ant}(\mathcal{P})$ given by $(F, G) \hat{\gamma}:=$ $(F \gamma, G \gamma)$, for $(F, G) \in \operatorname{Ant}(\mathcal{P})$, and $P \hat{\gamma}:=P$ is clearly an automorphism of $\operatorname{Ant}(\mathcal{P})$. Similarly, if $\mathcal{P}$ is a self-dual polytope and $\delta$ is a duality of $\mathcal{P}$, then $\hat{\delta}:(F, G) \mapsto(G \delta, F \delta)$ (and $P \hat{\delta}=P$ ) is also an automorphism of $\operatorname{Ant}(\mathcal{P})$. In other words, we have the following lemma. Keep in mind that we have defined the extended group of a non-self-dual polytope simply as its automorphism group.

Lemma 4.1. Let $\mathcal{P}$ be a polytope and let $\hat{\Gamma}(\mathcal{P})$ denote its extended group. Then, $\hat{\Gamma}(\mathcal{P})$ is (isomorphic to) a subgroup of $G(\operatorname{Ant}(\mathcal{P}))$.

It is not difficult to see that if $\psi: \mathcal{F}(A n t(\mathcal{P})) \rightarrow \mathcal{F}(\mathcal{P}) \times V$ is the bijection from Lemma 2.3, $\gamma \in \widehat{\Gamma}(\mathcal{P})$ and $\tilde{\gamma}$ is the automorphism of $\operatorname{Ant}(\mathcal{P})$ induced by $\gamma$, then for every flag $\Phi \in \mathcal{F}(\mathcal{P})$ and every $(n+1)$-tuple $v \in V$, we have that $(\Phi, v) \psi^{-1} \tilde{\gamma} \psi=(\Phi \gamma, v)$. This implies that if $\mathcal{P}$ is a self-dual polytope, dualities of $\mathcal{P}$ induce automorphisms of $\operatorname{Ant}(\mathcal{P})$.

The above observation, together with Lemmas 4.1 and 2.3 imply the following result.

Proposition 4.2. Let $\mathcal{P}$ an n-polytope and let $A n t(\mathcal{P})$ be its antiprism. If $\mathcal{P}$ is a $k$-orbit polytope, and $\operatorname{Ant}(\mathcal{P})$ is an m-orbit polytope, then:

- if $\mathcal{P}$ is self-dual, then $m \leq k \cdot 2^{n}$, 
- if $\mathcal{P}$ is not self-dual, then $m \leq k \cdot 2^{n+1}$.

Observe that, by the isomorphism given in (2.4), the facets of $\operatorname{Ant}(\mathcal{P})$ can be seen as the join product of sections of $\mathcal{P}$. This means that, maybe with the exception of the facets $\left(F_{-1}, F_{-1}\right) \cong \mathcal{P}^{*}$ and $\left(F_{n}, F_{n}\right) \cong \mathcal{P}$, the facets of $\operatorname{Ant}(\mathcal{P})$ are not prime with respect to the join product. Whenever $\mathcal{P}$ is a prime polytope with respect to the join product, we can obtain a lot of information about $\Gamma(\operatorname{Ant}(\mathcal{P})$.

Proposition 4.3. Let $\mathcal{P}$ be a prime polytope with respect to the join product. Then, $\Gamma(\operatorname{Ant}(\mathcal{P})) \cong \hat{\Gamma}(\mathcal{P})$.

Proof. By Lemma 4.1 we only need to show that any automorphism of $A n t(\mathcal{P})$ is in fact induced by either an automorphism or a duality (if $\mathcal{P}$ is self-dual) of $\mathcal{P}$. In this proof we abuse notation and refer to a polytope that is prime with respect to the join product simply as a prime polytope.

As pointed out above, when $\mathcal{P}$ is a prime polytope, the only two facets of $\operatorname{Ant}(\mathcal{P})$ that are also prime are $\left(F_{-1}, F_{-1}\right)$ and $\left(F_{n}, F_{n}\right)$. This means that any automorphism $\alpha \in$ $\Gamma(\operatorname{Ant}(\mathcal{P}))$ either fixes both such faces or interchanges them (as they cannot be permuted with any other, or they would not be prime). It is then easy to see that if $\alpha$ fixes them, then it induces an automorphism of $\mathcal{P}$ and that if interchanges them, then $\mathcal{P}$ is self-dual and $\alpha$ induces a duality.

Propositions 4.2 and 4.3 immediately imply the following result.

Corollary 4.4. Let $\mathcal{P}$ an n-polytope that is prime with respect to the join product and let $\operatorname{Ant}(\mathcal{P})$ be its antiprism. If $\mathcal{P}$ is a $k$-orbit polytope, and $A n t(\mathcal{P})$ is an $m$-orbit polytope, then:

- if $\mathcal{P}$ is self-dual, then $m=k \cdot 2^{n}$,

- if $\mathcal{P}$ is not self-dual, then $m=k \cdot 2^{n+1}$.

Lemma 3.2, together with Propositions 3.1 and 4.3 and Theorem 1.2, give us all the necessary tools to compute the automorphism of the antiprism of any polytope.

Theorem 4.5. Let $\mathcal{P}=\mathcal{Q}_{1}^{m_{1}} \bowtie \mathcal{Q}_{2}^{m_{2}} \bowtie \cdots \bowtie \mathcal{Q}_{r}^{m_{r}}$, where each $\mathcal{Q}_{i}$ is a prime polytope with respect to the join product. Then

$$
\Gamma(\operatorname{Ant}(\mathcal{P}))=\Pi_{i=1}^{r}\left(\left(\hat{\Gamma}\left(\mathcal{Q}_{i}\right)\right)^{m_{i}} \rtimes S_{m_{i}}\right) .
$$

\section{ORCID iDs}

Isabel Hubard (D) https://orcid.org/0000-0002-0960-3671

\section{References}

[1] A. Björner, The antiprism fan of a convex polytope, Am. Math. Soc. 18 (1997).

[2] M. N. Broadie, A theorem about antiprisms, Linear Algebra Appl. 66 (1985), 99-111, doi:10. 1016/0024-3795(85)90127-2.

[3] M. Dobbins, Antiprismless, or: Reducing combinatorial equivalence to projective equivalence in realizability problems for polytopes, 2013, arXiv:1307.0071 [math.CO]. 
[4] I. Gleason and I. Hubard, Products of abstract polytopes, J. Comb. Theory, Ser. A 157 (2018), 287-320, doi:10.1016/j.jcta.2018.02.002.

[5] P. McMullen and E. Schulte, Abstract Regular Polytopes, volume 92 of Encycl. Math. Appl., Cambridge University Press,Cambridge, 2002, doi:10.1017/cbo9780511546686. 